\title{
Références
}

\section{L'apprentissage de la communication par les médecins: aspects conceptuels et méthodologiques d'une mission académique prioritare}

\author{
Bernard MILLETIE, 2, , Marie Thérèse LUSSIER ${ }^{, 2,4}$, Johanne GOUDREAU \&, , 5
}

\begin{abstract}
Messages-lés
- La sociéé réclame des médecins plus humains et qui communiquent mieux avec les patients. Les cliniciens expérimentés et les chercheurs des domaines de l'éducation médicale et de la communication soulignent le rôle central et obligatoire de la communication médecin-patient au cours de la démarche clinique pour assurer le succès des stratégies de diagnostic et de traitement. - Les travaux des récentes décennies ont permis d'identifier les facteurs-clefs de l'ensegnement et del'apprentis sage d'une communication professionnelle efficace dansle domaine de la santé. Cesderniers incluent : 1) un référentiel sur cette communication, sol ide et basé sur les données de la recherche; 2 ) la pratique répécée des habi letés de communication associéeà une rétroaction (feed-back) spédifiqueimmédiate; 3) un cursusà la communication médecin-patient intégrétout au long des études médicales; 4) l'éval uation de l'acquisition des habiletés de communication comme critère de promotion ou de certification ; 5) des activités de connaissance de soi permettant de reconnaître ses propres valeurs et émotions intervenant dans la relation médecin-patient ; 6) des diniciensenseignants qui soient des modè es de rôle d'une communication professionnelle en santé adéquate - Les travaux du groupe Calgary-Cambridge proposent un référentiel solide et détaillé sur les habiletés de communication requises au cours de la pratique médicale et représentent une base intéressante pour élaborer des programmes de formation pertinents et efficients • Les facultés de médecine francophones ont la responsabilité de former des médecins aptes à communiquer avec leurs patients, entre eux et avec les autres membres de l'équi pe de soin. II et essentiel et urgent qu'elles mettent en place les stratégies propres à relever ce défi et à améliorer ainsi le résiltat des soins médicaux.
\end{abstract}

M ots clés Communication médecin-patient ; entrevue médicale ; approche centrée sur le patient ; éducation médicale; modalités d'apprentissage ; guide d'observation d'entrevue.

\begin{abstract}
Key Messages
- Soci ey calls for more humane physicians better able to communicate with patients Experienced dinicians and medical education and communication researchers underline the central and essential role of doctor-patient communication to the success of clinical processes such as diagnosis and treatment. - Research conducted during the last decades has allowed the identification of key factors of efficient teaching and learning health communication skills which include: 1) a solid, evidence based guide, of the communication skills to be mastered by health professionals, 2) frequent rehearsals and repetitive practice of these skillsassociated with immediateand specific feedback; 3) a longitudinal and integrated curriculum on doctor-patient communication all along medical training years; 4) communication skills assessment recognized as a formal promotion or certification criteria; 5) "self awareness » activities to help physicians become more conscious of their per sonal values and feelings that play a part in the doctor-patient relationships they engage in; 6) clinical teachers as adequate role models of effective health communication. - The Calgary-Cambridge initiative offers a solid and detailed guide of the communication skills required in medical practice. It thus represents an interesting framework for developing training programs for theseskills. • Francophone medical faculties and schools havethe responsibility for training physici ans as efficient communi cators not only with their patients but also with their medical colleagues as well as with other members of the health care team. It is essential and urgent that they deploy teaching strategiesin order to take up this challenge and thus improve medical care outcomes
\end{abstract}

Keys Words D octor-patient communication; medical encounter; patient-centered approach; medical educa tion; learning approaches; encounter observation guide.

Pédagogie M édicale $2004 ; 5$ : 110-126 


\section{Introduction}

Plusieurs organismes, associations ou institutions critiquent la performance des médecins au point de vue de la communication et réclament la mise en place de programmes de formation permettant une rédle acquisition des habiletés requises ${ }^{1-4}$. De plus, les recherches sur la communication médecin-patient ont grandement progressé au cours des dernières décennies. Ainsi les éléments communicationnels et relationnels nécessaires à la conduite d'une entrevue médicale efficace sont mieux connus et font consensus parmi les experts du domaine ${ }^{3,5-9}$. Par ailleurs, bien que certaines personnes puissent posséder une facilité « naturelle » à communiquer, on sait maintenant que les habiletés requises pour une communication professionnelle efficace ne sont pas innées et doivent être apprises $^{10-12}$. Or, chaque jour, les médecins doivent communiquer avec des patients et leur famille ainsi qu'avec plusieurs autres intervenants (collègues, professionnels de différentes disciplines, gestionnai res, etc). Ces différentes facettes de la communication sont souvent négligées au cours de la formation médicale. Comme beaucoup d'autres, nous croyons que l'acquisition d'une compétence en communication par les médecinsn'est pas qu'un «enrichissement agréable » de leur pratique mais qu'elle constitue une composante essentielle de la compétence clinique globale. Enfin, il est important de ne pas confondre l'enseignement de la communication médecin-patient avec celui de la psychologie médicale ou celui de la psychiatrie $\mathrm{C}$ es derniers sont parfois perçus comme assurant indirectement l'excellence en communication. $0 \mathrm{r}$, ces domaines sont différents et nécessitent des activités d'apprentissage et des modalités d'évaluation distinctes.

Le but du présent article est d'aider les responsables d'enseignement et les cliniciens superviseurs à planifier des programmes de formation à la communication et à l'entrevue médicale qui aient un réd impact sur la qualité des habiletés de communication des médecins et sur le résultat des soins médicaux. Pour ce faire, en se basant sur les résultats de recherche et l'expertise accumulée dans ce domaine, les auteurs identifient et décrivent les principes, contenus, formats et stratégies reconnus comme les plus aptes à faciliter le développement de cursus pertinents et la réussite de leur implantation.

\section{Problématique de l'apprentissage dela communication en médecine: les apports des « données probantes »}

D 'une façon générale, et même si la traduction française de la locution « evidence based medicine » soulève parfois des désaccords, le concept de « médecine fondée sur les données probantes » insiste pour que les décisions cliniques soient prises en tenant compte des meilleures preuves scientifiques disponibles ${ }^{13,14}$.

Les sciences de l'éducation constituent une discipline spé cifique où de nombreuses activités de recherche sont effectuées pour éclairer les enseignants sur les stratégies les plus susceptibles de favoriser le fait ... « que leurs étudiants apprennent » ! $0 r$, dans les milieux de formation médicale, plusieurs des méthodes pédagogiques utilisées semblent faire fi des acquis de la recherche en sciences de l'éducation, notamment lorsqu'il s'agit de l'enseignement de la communication.

A l'invitation de Van der Vleuten, expert reconnu en pédagogie médicale, soyons aussi exigeants en matière d'éducation médicaleet de formation cliniqueque nous le sommes quand il sagit du contenu biomédical de notre pratique clinique ${ }^{15}$.

Il est admis que la première étape de la planification d'une démarche d'apprentissage consiste à définir précisément les tâches concrètes que l'apprenant devra accomplir lorsqu'il exercera sa profession, ceci afin de bien identifier les compétences spécifiques qu'il doit acquérir ${ }^{16}$. O r, l'analyse des tâches du médecin conduit à constater que ce dernier effectue plusieurs milliers d'entrevues durant sa carrière ${ }^{7,17}$. De plus, ces entrevues constituent son principal instrument detravail ; elles lui permettent de développer le processus de raisonnement clinique, de planifier les investigations diagnostiques et de prendredes décisions concernant

1. Cinique de médecine familiale, Cité de la Santé de Laval, 1755, bld René Laennec, Laval (Québec), Canada, HTM 3L9, Tél. : (450) 975-5355, mailto:bernard.millette@umontreal.ca

2. Département de médecine familiale, Faculté de médecine, Université de Montréa.

3. Faculté des sciences infirmières, Université de Montréal

4. Équipe de recherche en soins de première ligne, Oté de la Santé de Laval.

5. Unité de recherche et de développement en éducation des sciences de la santé (URDESS), Faculté de médecine, Université de Montréa - Correspondance : Bernard Millette adresse ci-dessus. 


\section{Références}

les stratégies thérapeutiques; elles sont le moyen de fournir aux patients l'information pertinente et de développer avec eux des liens essentiels de collaboration. Pourtant, malgré ces faits évidents et connus, l'apprentissage de la communication médecin-patient demeure souvent le parent pauvre des cursus médicaux. En effet, la plupart du temps, cet apprentissage est effectué: 1) sans qu'il soit fait référence à un modèle étayé par un cadre conceptuel solide ; 2) en dehors d'une approche pédagogique rigoureuse basée sur les résultats de recherche dans les champs de l'éducation et de la communication et : 3) en saffranchissant, pour les habiletés visées, d'une obligation de résultat, qui soit attestée par une évaluation « sanctionnelle » fiableet valide

\section{L'acquisition des habiletés de communication médecin-patient}

L'apprentissage des habiletés de communication exige l'acquisition d'un savoir, d'un savoir-faire et d'un savoir-être spécifiques et complexes. Ces apprentissages impliquent dès lors un enseignement basé sur des méthodes pédagogiques complexes. La psychologie cognitive offre un éclairage intéressant sur l'acquisition des habiletés de communication. En effet, les recherches suggèrent que les nouvelles connaissances sont apprises et retenues en se liant aux réseaux des savoi rs déjà ancrés dans la mémoire à long terme ${ }^{18,19}$. C'est pourquoi, selon ce modèle, il est essentiel d'activer les connaissances déjà acqui ses et les liens déjà en place pour favoriser l'acquisition de nouvelles connaissances. Sous certaines conditions, le travail en petit groupe est susceptible de faciliter cette réactivation en permettant l'émergence et l'interaction des «théories naiives » des apprenants face à un nouveau problème et en orientant l'intégration des nouvel les informations nécessaires à I'aide du processus d'analyse et de résolution du problème soumis. Sous certaines conditions, liées à la nature de la tâche d'apprentissage proposée, le travail en petit groupe peut également participer à une « contextuali sation » plus authentique des connaissances et favoriser leur réutilisation ultérieure en situation clinique.

II est aussi admis que la discussion entre pairs a une influence sur les changements d'attitude ${ }^{20,21}$. Enfin, selon Kurtz et Smith ${ }^{10,11,22}$, la démonstration et la pratiquerépé tée faciliteraient l'apprentissage des habiletés de communication et renforceraient la capacité de les utiliser lorsqu'elles sont requises. Les programmes de formation à la communication doivent donc inclure des activités per- mettant un apprentissage, intégré et contextuel, des connaissances, des attitudes et des habiletés nécessaires pour communiquer efficacement.

En cohérence avec les données précédentes et en se fondant sur une revue extensive de la littérature scientifique de langue anglaise consacrée aux activités d'enseignement et d'apprentissage de la communication médecin-patient, Aspegren ${ }^{23}$ note que les méthodes d'apprentissage expé rientielles, liées à la solution de problèmes cliniques et incluant une rétroaction (fæed-back) immédiate, sont les plus efficientes. Par ailleurs, plusieurs études établissent que les habiletés de communication peuvent s'enseigner et s'apprendre mais aussi qu'elles peuvent décliner, si elles ne sont pas réactivées ${ }^{23-25}$. II est toutefois difficile de statuer, à partir de la littérature, sur l'intensité et la durée idéales des activités d'apprentissage à la communication médecinpatient. II parait toutefois assez probable que l'impact d'une seule ou de quelques brèves sessions de formation (une à quel ques heures) non répétées soit quasi-nul.

\section{Les éléments essentiels pour l'apprentissage de la communication médecin-patient}

Les données de la recherche et l'opinion des experts du domaine permettent de suggérer que les six éléments suivants devraient être inclus dans une stratégie de formation visant l'apprentissage réussi de la communication professionnelle par les médecins $\boldsymbol{S}^{10,26,27}$ (Tableau 1 ) : 1) le recours à un référentiel spécifique (établi à partir des résultats de la recherche et identifiant clairement les apprentissages à acquérir) utilisé comme facteur de structure et de cohérence de l'enseignement tout au long du cursus ; 2) une pratique répétée des nouveaux apprentissages, incluant une rétroaction (feed-back) immédiate et spécifique sur la performance, ceci afin de favoriser l'amélioration, la consolidation et l'internalisation des acquis; 3) un cursus de formation cohérent et longitudinal sétendant du début des études médicales initiales aux années de résidanat (quelle que soit la discipline clinique) et influençant la formation médicale continue ; 4) la mise en place d'un processus formel d'évaluation de l'acquisition par les apprenants des compétences visées ; 5) des activités de " découverte de soi ", afin que les médecins soient plus conscients des valeurs, croyances et émotions qui influencent leurs comportements pendant une entrevue ; 6 ) le côtoiement, par les apprenants, decliniciens qui soient des modèles de rôle explicites d'une communication médecin-patient de qualité, cohérente avec les enseignements reçus. 


\section{Tableau 1 : Les éléments d'une stratégie efficace pour l'enseignement et l'apprentissage de la communication}

1. Un référentiel basé sur les données scientifiques probantes

2. Une pratique des habiletés avec une rétroaction spécifique

3. Un cursus cohérent et longitudinal

4. Un processus d'évaluation des apprentissages

5. Des activités de « découverte de soi »

6. Des cliniciens, modèles de rôle

\section{Le référentiel}

Plusieurs auteurs ont marquéle domaine de la communication médecin-patient au cours des dernières décennies $7,8,10,12,28 \cdot 33$. On peut retenir en particulier queBird et Cohen-Cole ${ }^{34}$, puis Lazare et al. ${ }^{35}$, ont proposé, pour l'entrevue médicale, un modèle à trois fonctions qui, briè vement résumées, sont : 1) la collecte des données nécessaires; 2) le développement dela relation médecin-patient et 3) l'information et la motivation du patient. Leur modèle s'appuie sur une perspective dynamique de l'entrevue et sur une attention aux processus s'y déroulant. Ces auteurs mettent ainsi l'accent sur les fonctions géné riques de toute entrevue, qu'elle se passe au chevet du patient hospitalisé, au service des urgences, en clinique externe, en cabinet ou à domicile. C ette façon de conceptualiser l'entrevue, en termes de fonctions, diffère de la perspective traditionnelle où elle est abordée sous l'angle de sa structure.

Par ailleurs, il est essentiel, lors de toute démarche clinique, de concilier et d'intégrer le raisonnement clinique et la communication 12,1433,36. D ans ce contexte, l'acquisition réussie des habiletés de communication est un élé ment incontournable. En prenant appui sur lemodèledes trois fonctions de l'entrevue et sur les données probantes issues des recherches en communication et en pédagogie, Kurtz et ses collaborateurs proposent en l'occurrence un modèle intégrant la communication à la démarche clinique $e^{810-12,37}$. Ces auteurs ont élaboré un guide des comportements et tâches observables faisant partie d'un processus de communication efficace : le Guide CalgaryCambridge (Figure 1 et annexe 1). Ils proposent une démarche prêtant une attention aussi vive aux éléments factuels à découvrir (le contenu) qu'aux habiletés à utiliser (le processus) (voir figure 2). Les travaux de Kurtz et al. ${ }^{10}$ constituent une excellente source et une base solide pour l'élaboration d'un cursus de formation à la communication médecin-patient et leur guide peut être utilisé comme modèle de référence pour la pratique et l'enseignement de l'entrevue médicale. Les qualités de ce modèle sont, à nos yeux, sa rigueur scientifique, la clarté de sa démarche, sa pertinence clinique et son souci pédagogique; il intègre, en outre, l'ensemble des ééments d'une approche biopsychosociale de l'entrevue médicale.

Selon les auteurs du guide Calgary-Cambridge, les apprentissages souhaités ainsi que la logique qui les soustend doivent être clairs pour les étudiants et les enseignants. C'est pourquoi les éléments théoriques de leur modèle doivent être intégrés à la formation. De brèves présentations magistrales et/ou la distribution de documents écrits peuvent être utilisées, tout en se rappelant que la manipulation des concepts par les apprenants (analyse et résolution de problèmes, discussion en petits groupes, travaux individuels) et leur application à des situations cliniques usuelles (pratique concrète) facilitent un meilleur et plus utile enchâssement en mémoire ${ }^{15,19}$.

\section{La pratique, la rétroaction... La pratique, la rétroaction... La pratique, la rétroaction...}

II ne viendrait à aucun clinicien l'idée de se contenter d'exercices sur papier ou encore de la réal isation d'un seul essai pour reconnaitrel'aptitude à effectuer de façon autonome des appendicectomies et, partant, d'autres chirurgies (colectomie... ).

D ans cet ordre d'idée, l'apprentissage de la communication en tant qu'habileté complexe (savoir, savoir faire et savoir être) exige plusieurs mises en pratique dans un contexte proche du vécu professionnel futur. D e plus, en se basant sur les données de la recherche en éducation, Kurtz et ses collaborateurs soutiennent qu'il est essentiel d'allier la pratique à une rétroaction immédiate et spécifique $^{10}$.

Certes, les leçons magistrales et les exercices sur papier peuvent être des éléments concourant à la transmission et, possiblement, à la rétention de certains savoirs utiles. Toutefois ces méthodes d'enseignement ne garantissent nullement I'application de ces savoirs en situation clinique réelle. Par ailleurs, les habitudes acquises lors des années de formation, qu'elles soient bonnes ou mauvaises, se 


\section{Références}

\section{Figure 1 : Le Guide Calgary-Cambridge de l'entrevue médicale ${ }^{12}$ (texte traduit de l'anglais)}

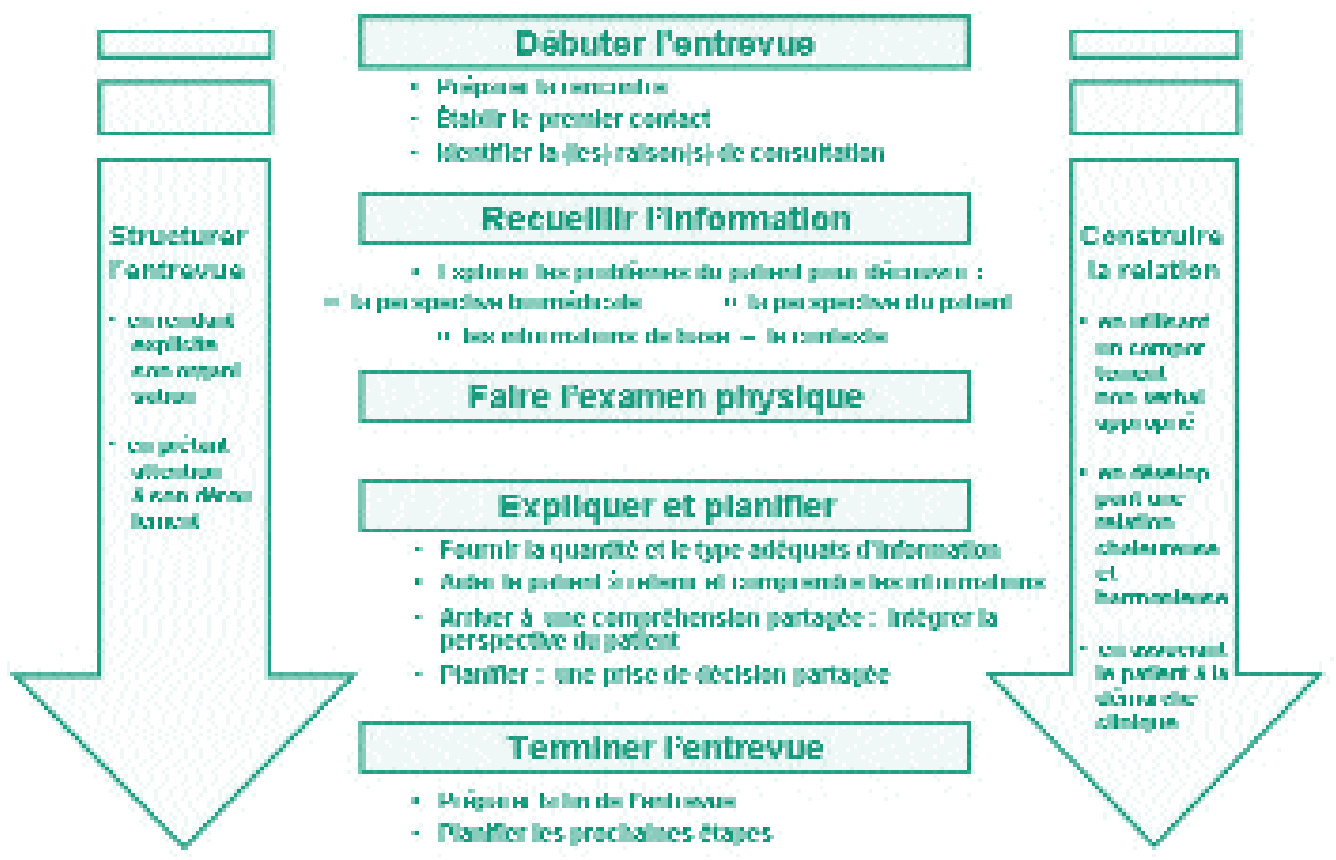

Figure 2 : Les liens « contenu et processus » lors d'une entrevue, une illustration à l'aide de la tâche : recueillir l'information ${ }^{12}$ (texte traduit de l'anglais)

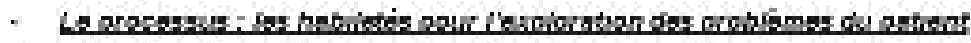

\section{LVlistale da patien}

Le stece des questions: Ac oantries s fermies

- ISianis xalion

Lafactitation

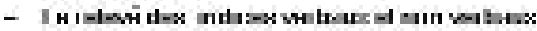

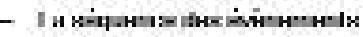

Les resumes

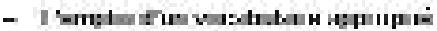

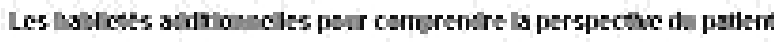

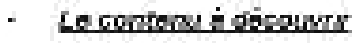

Lx perspective bomedicole (la malsdie)

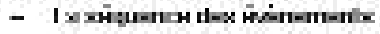

- I vestene the somgdimes

La resic perthente des systemes

Lx perspective du patient [es malases]

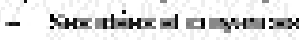

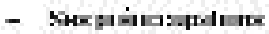

Ses attectes

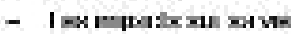

Ses tmotiens

Les informations de base - le centanta

Lhistore meiteale atherieure

Lhistore of prise de melcancets et ies aleriles

- I mx uelícistels lemtrar

Les artecessats persoints et soclaux

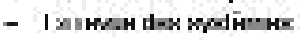


consolident avec letempset ne peuvent êtremodifiées que par des stratégies d'apprentissage spécifiques, donc grâce à des stratégies d'enseignement tout aussi spécifiques incluant notamment des rétroactions répétées (føed-back). II a aussi été démontré que les acquis des premières années d'études peuvent samenuiser sils ne sont pas réactual isés et renforcés au cours des formations cliniques (externat, internat ou résidanat). C'est d'ailleurs pour cette raison quel'industrie aéronautique oblige ses pilotes à des simulations fréquentes et répétées de vol reprodui sant des situations de routine, des incidents mineurs et d'autres, catastrophiques. II est tout aussi essentiel en médecine de pré voir, tout au long de la formation et durant la vie professionnelle, des périodes d'apprentissage et de consolidation des habiletés de communication qui incluent des mises en application répétées associées à des rétroactions immé diates.

\section{Un cursus de formation cohérent et longitudinal}

Les éléments rapportés dans le paragraphe précédent militent, à eux seuls, pour la mise en place d'un cursus à la communication qui soit intégré aux autres ééments de la formation médicale et qui se poursuive longitudinalement tout au long de la formation de l'étudiant, quelle que soit sa discipline « finale ».

$M$ ais, le point majeur de l'argumentaire est qu'une communication médecin-patient adéquate est essentielle dans tous les champs disciplinai res de la médecine. Les institutions d'enseignement et les organismes de développement professionnel continu ont donc la responsabilité d'élaborer, conjointement, une stratégie propre à assurer, tout au long de la période de formation des étudiants puis de la carrière des cliniciens, les apprentissages pertinents ainsi que leur actualisation et leur renforcement.

Bien que d'autres professionnels (psychologues, travailleurs sociaux, ... ) puissent intervenir dans la formation à la communication, il est essentiel que les enseignants principaux soient des médecins (de disciplines variées) et, au niveau del'internat ou du résidanat, des médecins de la discipline clinique des apprenants.

II est à noter que la qualité de la formation dispensée en communication médecin-patient constitue maintenant un élément majeur évalué lors des processus d'accréditation des facultés de médecine et des organismes de formation continue de plusieurs pays $5^{2,38,39}$.

\section{L'évaluation formelle des apprentissages}

L'évaluation « sanctionnelle » (ou sommative) des habiletés de communication varie en profondeur et en qualité selon les milieux d'enseignement. Dans certaines institutions, les étudiants ne sont évalués que sur leur connaissance déclarative des éléments d'une communication médecin-patient adéquate, l'évaluation de leurs habiletés, de leurs attitudes et de leurs performances dans ce domaineétant totalement lai ssée de côté. Ainsi, dans ces milieux, le message implicite est clair : les habiletés de communication médecin-patient sont des éléments importants, relevant plus ou moins du talent « inné » des personnes, qu'il est «agréable», mais non essentiel, de posséder. Or, la réal ité accrédite tout le contraire. Le message aux étudiants devrait donc être explicite et sans ambiguïté : I'obtention du diplôme de médecine et le droit d'exercer à titre de clinicien exigent l'acquisition des habiletés de communication.

L'évaluation des connaissances et des habiletés de communication peut être effectuée, lors d'entrevues réelles ou simulées, à l'ai de d'instruments fidèles et valides. Comme lementionnait M iller ${ }^{40}$, les étudiants, lors des évaluations, ne doivent pas uniquement êtretenus de confirmer qu'ils " savent » et qu'ils « savent comment » mais surtout de "montrer comment ils font ", c'est-à-dire comment ils agissent en pratique. $\mathrm{N}$ ous reviendrons plus loin dans le texte sur le thème de l'évaluation.

\section{Des activités de « découverte de soi »}

N orbert Bensaid écrivait : «Peu importe qu'un trouble ait une origine psychique ou somatique; de toutes façons la relation entrelemédedin es son malade va déaider decequedeviendra la maladie, de la manière dont elle sera vécue, interprétée, soigné, guérie $»^{41}$. La relation médecin-patient n'est pas seulement tributaire des habiletés de communication ; elle est influencée par des facteurs individuels inhérents au médecin et au patient, comme la personnalité, I'histoire personnelle (enfance, liens avec les parents, orientation sexuelle, vie de couple... ), la culture, la religion, les valeurs, etc $20,26,41,42$.

C'est pourquoi nous estimons essentiel que les médecins se connaissent mieux eux-mêmes. A notre avis, cette connaissance de soi consiste principalement à pouvoir identifier et discuter ses propres valeurs, croyances, attitudes et façons d'agir et de réagir. Elle favorisel'ouverture aux autres qui est nécessaire pour collecter et interpréter les informations utiles à la démarche clinique et pour proposer des 


\section{Références}

conduites thérapeutiques efficaces. Globalement, cette ouverture se reflète par une attitude de respect et decuriosité pour la « réalité » et les croyances de chaque patient ${ }^{43}$.

L'apprentissage de la communication devrait donc inclure des activités de «découverte de soi » venant enrichir celles qui visent l'acquisition des habiletés de communication, sans toutefois $s^{\prime} y$ substituer. Plusieurs activités éducatives ont été proposées par différents auteurs pour favoriser la " découverte de soi ». N ous ne ferons ici qu'en mentionner quelques-unes, invitant les lecteurs à consulter la litté rature publiée sur ce sujet 20,26,33,41,44.46 : réflexions individuelles sur ses propres activités (portfolio); observations critiques de ses entrevues (audio, vidéo), rencontres en petits groupes (groupes Balint, groupes de paroles, groupes de partage sur sa famille d'origineet sa culture... ), cursus spécifiques incluant des discussions sur des thèmes tels que les influences familiales, les patients difficiles, l'équilibre carrière professionnelle/vie privée, etc. Certains étudiants ou praticiens choisiront parfois une démarche personnelle comme la psychothérapie.

Q uelle que soit l'activité choisie, celle-ci doit chercher à favoriser la prise de conscience des facteurs personnels qui influencent la perception, l'interprétation et l'action des médecins lorsqu'ils rencontrent des patients. II ne s'agit pas de tenter d'éliminer les émotions des médecins, ni de faire fi des valeurs auxquelles ils tiennent mais de les rendre plus conscients de l'influence de ces facteurs afin d'éviter que ceux-ci interviennent de façon inappropriée dans la communication médecin-patient et dans les décisions cliniques.

\section{Des cliniciens, communicateurs modèles}

Selon $\mathrm{H}$ afferty ${ }^{41}$, le modèle de rôle constitue un moteur très puissant pour l'apprentissage d'habiletés telle que la communication. Ainsi, lesfuturs médecins sont fortement influencés par ce qu'ils observent chez leurs superviseurs. C'est pourquoi la responsabilité des activités visant l'apprentissage des habiletés de communication médecinpatient ne peut reposer uniquement sur un nombre limité d'enseignants d'une faculté, ni être confiée exclusivement à des enseignants non médecins. En effet, comme on peut l'observer dans plusieurs milieux de formation, ces situations conduisent à un clivage entre la pratique clinique et la pratique de la communication et, ai nsi, à la perception que la maitrise des aspects bio-médicaux est indépendante de la maîtrise de la communication qui, elle, serait accessoire. Les conséquences d'un tel clivage sont néfastes.

Ainsi, les activités d'enseignement et d'apprentissage réalisées individuellement ou en petits groupes doivent obliga toirement être consolidées et renforcées lors des différentes activités cliniques. Les facultés de médecine ne réussiront pas à former d'excellents communicateurs si, suite à un atelier sur l'exploration respectueuse de la perspective du malade, les étudiants en stage dans un service clinique y voient leurs «patrons s diriger des entrevues sans lai sser de place aux patients pour intervenir ou exprimer leurs craintes, leurs croyances ou leurs attentes. De façon similaire, les milieux de formation ne parviendront pas à faire accepter le message que la communication est une compétence médicale incontournable si leurs étudiants assistent à des sessions de formation à la communication où les médecins enseignants sont absents ou n'y jouent qu'un rôle secondaire II est donc impératif que chaque faculté de médecine, chaque réseau hospitalo-universitaire, chaque milieu ambulatoire d'enseignement, initie ou consolide des activités explicitement dédiées au renforcement des habilités de communication des cliniciens qui y oeuvrent et propres à leur fournir un cadre conceptuel pour leurs discussions avec les stagiaires au sujet de la communication médecin-patient. De plus, des stratégies devraient être mises en place pour faciliter l'émergence, parmi les cliniciens, de personnes ressources pour l'enseignement de la communication professionnell $\mathrm{e}^{10,47,48}$.

\section{Des exemples d'activités d'enseignement à la communication}

Actuellement dans le réseau académique de la faculté de médecine de l'U niversité de M ontréal, les étudiants profitent d'activités d'apprentissage inspirées des travaux du groupe $C$ algary-C ambridge

Les principaux moyens de formation utilisés sont : 1) la supervision individuelle d'entrevues ; 2) les ateliers en petit groupe et : 3) dans quelques milieux, la tenue de «groupes Balint ». Au cours des deux premières années des études médicales initiales, dans le cadre des cours «Introduction à la médecine clinique » et «Initiation à la démarche clinique », plusieurs heures d'ateliers sont consacrées à l'apprentissage de l'histoire de cas et de l'examen physique. A ce niveau, l'accent est surtout mis sur les éléments du guide Calgary-C ambridge touchant I'amorce de l'entrevue et le recueil des données cliniques, tout en portant une attention à la structure générale de l'entretien 
et à la nécessité de construire la relation. D urant le résidanat de médecine de famille (médecine générale), I'approche varie selon les sites de formation mais tous offrent des activités sur l'entrevue.

A la Cité de la Santé de Laval (I'un des neuf milieux de formation du résidanat de médecine de famille de I'U niversité de Montréal), un total d'environ 40 à 60 heures est spécifiquement disponible pour chaque résident au cours de ses deux années de formation, individuellement ou en groupe, pour développer et consolider ses habiletés d'entrevue et discuter d'enjeux individuels touchant la communication.

II faut ajouter à cet effort le temps dédié à la discussion des difficultés de communication médecin-patient à l'occasion du travail clinique quotidien effectué par les résidents.

\section{Les séminaires d'entrevues médicales (SEM) à la Cité de la Santé de Laval}

Au Q uébec en 2004, le programme de résidanat en médecine de famille (l'équivalent de l'actuel troisième cycle de médecinegénéral eet du futur Diplôme d'études spécial isées (DES) de médecine générale en France) est dedeux ans. A M ontréal, les résidents effectuent six mois de stage concentrés en médecine de famille (trois mois, chaque année du résidanat), tout en suivant leur clientèle sur une base hebdomadaire tout au cours de leur formation. C haque semaine, durant les six mois de stage à la clinique de médecine de famille, une demi-journé est consacrée à l'apprentissage de la communication. Le séminaire d'entrevues médicales (SEM) est divisé en deux parties : le SEM -atelier d'une durée de deux heures et le SEM -vidéo d'une durée d'une heure. Durant ces activités, une attention particulière est portée à la création d'un climat de collaboration où il est permis aux participants d'essayer, de se tromper, de réessayer et ainsi de suite. Une documentation de base est remise peu avant ou lors de la première participation des étudiants aux SEM . Elle inclut le guide Calgary-C ambridge, les schémas qui y sont associés, un court texte sur les principales stratégies de communication ainsi qu'un codex d'articles publiés par un des auteurs (M TL en collaboration avec Claude Richard, un chercheur de l'équipe de la Cité de la Santé). De plus, selon les sujets abordés, d'autres textes peuvent être distribués lors d'un SEM spécifique.

\section{Le SE M -atelier}

Tous les externes (en France, étudiants en 4 e année du deuxième cycle des études médicales [DCEM 4]) et résdents en stage à la clinique de médecine de famille participent à cette activité, ce qui représente un groupe variant de 5 à 10 personnes. En sappuyant sur le modèle « démonstration-pratique rétroaction ${ }^{21}$ et en tenant compte de la nécessitéd'accroître la motivation des étudiants, chaque session du SEM-atelier priorise un thème, c'est-à-dire une tâche, unehabileté ou unesituation (par exemple: explorer la perspective d'un patient, exprimer de l'empathie, accueillir un patient en colère.. ). L'activité débute par une discussion des difficultés rencontrées ou appréhendées par les participants en regard du thème choisi.

Cette discussion peut être initiée par : 1) une question ouverte; 2) une vidéo illustrant une situation pour laquelle les habiletés ou tâches à apprendre sont nécessaires ou : 3) une mise en situation sous forme d'un jeu de rôle. Les objectifs d'apprentissage de l'atelier sont alors clarifiés et les tâches ou habiletés de communication visées sont décrites puis illustrées à l'aide d'une démonstration réalisée en direct ou préenregistrée sur bande vidéo (entre vue simulée ou entretien avec un « vrai » patient). C ette partie de l'atelier peut durer de 20 à 40 minutes.

Une fois les tâches ou habiletés clarifiées, une ou plusieurs simulations sont utilisées pour en permettre l'apprentissage. Les étudiants sont invités, chacun à leur tour, à jouer le rôle du médecin ou du patient. Lorsqu'ils ne jouent pas de rôle, ils reçoivent la consigne : 1) d'observer l'entrevue simulée (en se référant au guide Calgary-Cambridge) ; 2) d'y identifier spécifiquement les éléments facilitant la communication ainsi que ceux qui semblent créer des difficultés et : 3 ) de suggérer comment on pourrait faire autrement.

Chaque jeu de rôle dure environ dix minutes. Les protagonistes sont observés directement dans la même pièce ou par le biais d'un système audio-visuel. Lors de ces exercices, les règles de base de l'utilisation du jeu de rôle sont appliquées ${ }^{10,49,50}$. Ainsi, après sa prestation et avant tout autre intervention, l'étudiant ayant joué le rôle du médecin est invitéà exprimer ses propres commentaires, à identifier les actions qui ont facilitél'entretien médical et celles qui auraient pu être conduites différemment. II peut également identifier les éléments pour lesquels il souhaite des suggestions de la part des participants. Puis, l'étudiant ayant joué le rôle du patient émet ses commentaires, suivi par les observateurs. 


\section{Références}

Les commentaires offerts par chacun, apprenants et animateurs, doivent répondre aux canons de la rétroaction tels qu'ils sont décrits dans la littérature $e^{10,29,50.52}$. Ainsi, une rétroaction (fæedback) constructive doit :

1) porter sur des éléments convenus à l'avance avec l'étudiant qui joue le rôle du médecin et/ou par les animateurs ;

2) être spécifique, c'est-à-dire cibler des comportements observés et modifiables ;

3) être équilibrée, mettant en évidence les éléments positifs autant que ceux à améliorer ;

4) comporter des suggestions concrètes et réalistes d'amélioration (ainsi on demande à celui qui fait des suggestions d'en faire la démonstration).

Suite à la discussion, un autre jeu de rôle (le même cas ou une situation différente) est exécuté. Les scénarios de la plupart des jeux de rôle sont rédigés avant les ateliers afin de s'assurer qu'ils permettent d'atteindre les objectifs visés. Toutefois, l'approche demeure souple et il arrive que des jeux de rôle soient improvisés sur place afin de répondre à des besoins d'apprentissage identifiés en séance.

L'animateur du SEM centre les discussions sur les comportements observés. II insiste sur l'emploi d'un vocabulaire approprié (basé sur le guide CalgaryCambridge) pour décrire ce qui a été vu et cequi pourrait être fait. En général, il évite les longs échanges ou débats qui font rater aux étudiants d'excellentes occasions d'apprendre par une pratique supervisée répétée. II demeure attentif au climat affectif tout au long du SEM et intervient, en groupe ou individuellement, Iorsque cela est opportun. C ette partie de l'activité dure environ de 70 à 90 minutes.

Les dernières dix minutes servent à résumer les apprentissages, à faire ressortir les messages-clefs et à déterminer les points non résolus qui pourraient faire l'objet de tâches à réaliser avant ou lors de la prochaine rencontre. A la fin de la séance, l'animateur complète une feuille de suivi identifiant les thèmes abordés et les rôles joués. II complète le sommaire en inscrivant les ap prentissages couverts (en référence au guide $C$ algary-Cambridge). Ces données sont conservées dans le registre des SEM . Le thème suivant est décidé en tenant compte des suggestions des participants.

\section{Le SEM -vidéo}

Suite au SEM -atelier, chaque résident, à tour de rôle selon une liste préétablie, est invité à discuter avec un des animateurs une ou des entrevues qu'il a préalable ment enregistrées sur une bande magnétoscopique. En accord avec les données de la littérature concernant ce type d'activité, il est demandé au résident de choisir les extraits de la bande vidéo qu'il souhaite discuter, de procéder à une autoévaluation de sa performance à l'aide du guide Calgary-Cambridge et de clarifier ses attentes ${ }^{7}$ 10, 53. Le contrôle technique est laissé à l'étudiant et une entente est faite sur les règles d'interruption. Les commentaires du médecin-animateur sont adaptés à la situation tout en se référant au guide Calgary-Cambridge et aux principales stratégies de communication. Un résumé des messages-clefs est conjointement effectué à la fin de la rencontre. Ces activités sont aussi consignées dans le registre des SE M.

\section{Le choix des thèmes des séminaires d'entrevues médicales (SEM-atelier)}

Sanson-Fisher et Cockburn ${ }^{54}$ suggèrent sept critères pertinents pour choisir les situations ou les problématiques cliniques devant être ciblées pour les cours et les apprentissages à la communication. Selon ces auteurs, les éléments suivants doivent être réunis : 1) la situation retenue est fréquemment rencontrée ; 2) elle entraîne, ou a le potentiel d'entraîner, un lourd fardeau de malaises et de morbidité ; 3 ) des données probantes existent en faveur de la nécessité d'améliorer les habilités des médecins pour faire face à cette situation ; 4) I'intervention, dont les habilités de communication sont une ou la composante principale, a été évaluée comme étant efficace pour cette situation ; 5) l'intervention offre un ratio coûts/bénéfices favorable et : 6 et 7) l'intervention proposée est jugée acceptable (pertinence, faisabilité, utilité) autant par les médecins que par les patients. Rappelons qu'il est essentiel d'éviter le clivage « démarche clinique/habiletés de communication ». U n des pièges des ateliers sur la communication est de choisir des situations extrêmes (trouble sévère de personnalité; famille très dysfonctionnelle...) plutôt que de se centrer sur les problèmes usuels rencontrés dans une pratique médicale courante. Le tableau 2 présente différents exemples de mises en situation utilisées dans notre milieu, en fonction des thèmes ou tâches visés par les ateliers. 


\begin{tabular}{|c|c|}
\hline Thèmes ou tâches de l'atelier & Situations cliniques pour les aborder \\
\hline Le survol des raisons de consultation & $\begin{array}{l}\text { Le médecin veut aborder diabète et lipides; } \\
\text { le patient : parler de son arthral gie, sa fatigue, } \\
\text { son lecteur de glycémie. }\end{array}$ \\
\hline $\begin{array}{l}\text { La notion des agendas (objectifs) } \\
\text { du patient / du médecin }\end{array}$ & $\begin{array}{l}\text { Le médecin veut prescrire un médicament pour l'hypertension } \\
\text { Le patient hypertendu se sent bien, veut des conseils } \\
\text { «naturels » et craint le «chimique». }\end{array}$ \\
\hline L'exploration de la perspective du patient & $\begin{array}{l}\text { Patient avec un lymphome (stade précoce) } \\
\text { qui refuse la chimiothérapie. }\end{array}$ \\
\hline Le soutien à l'observance & $\begin{array}{l}\text { Le patient reçoit une première ordonnance } \\
\text { pour traiter son taux de cholestérol élevé. }\end{array}$ \\
\hline La gestion des émotions & $\begin{array}{l}\text { Un patient est nettement en colère au sujet du retrait médical } \\
\text { de son permis de conduire une automobile. }\end{array}$ \\
\hline L'annonce d'une mauvaise nouvelle & $\begin{array}{l}\text { Le médecin doit informer une patiente du résultat } \\
\text { positif de sa biopsie du sein (cancer). }\end{array}$ \\
\hline L'entrevue à plusieurs & $\begin{array}{l}\text { Une « G rand-maman » diabétique est « amenée » } \\
\text { par sa fille chez le médecin. }\end{array}$ \\
\hline L'entrevue en présence d'un interprète & $\begin{array}{l}\text { Un patient asiatique fatigué voit le médecin } \\
\text { avec l'aide d'un parent interprète. }\end{array}$ \\
\hline D ire non à un patient & $\begin{array}{l}\text { Le patient croit nécessaire un arrêt de travail } \\
\text { que le médecin juge non justifié. }\end{array}$ \\
\hline Le dévoilement de soi & $\begin{array}{l}\text { Le patient demande à son médecin si elle est mariée, } \\
\text { combien elle a d'enfants... }\end{array}$ \\
\hline L'empathie & $\begin{array}{l}\text { Un patient consulte pour céphalée dans un contexte } \\
\text { de surcharge de travail et de stress familiaux. }\end{array}$ \\
\hline La prise de décision partagée & $\begin{array}{l}\text { Une femme consulte pour des symptômes liés à } \\
\text { la ménopause (hormonothérapie : oui ou non ?) }\end{array}$ \\
\hline Le patient multi-symptomatique & $\begin{array}{l}\text { Le médecin reçoit un patient se plaignant de multiples } \\
\text { symptômes laissant soupçonner un trouble de personnalité } \\
\text { ou un trouble somatoforme. }\end{array}$ \\
\hline
\end{tabular}

* Liste partielle des thèmes et s stuations abordés du rant leS SEM à la Cité de la Santé de Laval 


\section{Références}

Lors de tous les SEM, nous insistons pour que les discussions soient faites en lien avec les éléments du guide Calgary-C ambridge, ceci afin de permettre aux étudiants de se construire une « structure internal isée » de leurs stratégies de communication. Cette approche éducative vise à les aider à mieux enchâsser en mémoire les apprentissages du moment pour ainsi faciliter leur utilisation ultérieure.

\section{L'évaluation, un moteur incontournable de l'apprentissage de la communication}

\section{L'évaluation formative}

L'évaluation constitue un levier très puissant pour favoriser l'acquisition de compétences spécifiques. II est essentiel toutefois que l'évaluation porte sur les apprentissages recherchés. Pour demeurer cohérente avec le référentiel choisi, ici le guide $\mathrm{C}$ algary-Cambridge, l'évaluation des habiletés de communication doit porter sur la compétence avec laquelle les candidats, au cours de l'entrevue médicale, accomplissent les diffé rentes tâches identifiées dans ce guide. Lors des activités cliniques usuelles et des périodes formelles de formation, de multiples opportunités s'offrent pour effectuer, à l'aide du référentiel, des évaluations formatives, utiles aux apprenants.

\section{L'évaluation « sanctionnelle 》 (ou sommative)}

Les tâches réalisées au cours d'un entretien médical sont complexes et influencées par de nombreux facteurs. Ainsi, il semble que la performance en communication varie selon la nature des problèmes abordés au cours d'une entrevue ${ }^{55}$. Cela signifie, entre autres, que pour porter un jugement évaluatif sur la performance « usuelle » d'un apprenant, l'évaluation devrait inclure plusieurs entrevues touchant des problématiques variées. D e plus, bien que différents instruments d'évaluation de la communication aient été proposés au cours des années, les difficultés et les problèmes liés à leur utilisation n'ont pas tous été surmontés , $1,0,33,37,56-58$. Choisir et appliquer une modalité d'évaluation fidèle, valide et réalisable localement demeure un défi. Heureusement, certains guides comme les grilles SEGUE ${ }^{58}$ et $\mathrm{Calgary}-\mathrm{C}$ ambridge ${ }^{37}$ aident à réduire la difficulté en concentrant l'attention sur des items observables et, jusqu'à un certain point, mesurables.
D ansle cadre de ses examens de certification, le « Royal College of General Practitioners » du RoyaumeUni a développé un processus d'évaluation basé sur des entrevues effectuées par les candidats lors de leur travail clinique usuel ${ }^{59,60}$. Ainsi, plusieurs entrevues enregistrées sur bande magnétoscopique sont évaluées par un jury à l'aide d'une grille préétablie. Un minimum de huit entrevues différentes doivent être enregistrées dont au moins une impliquant un enfant de moins de 10 ans et une autre, incluant une dimension psychologique ou sociale importante. II est recommandé aux candidats d'enregistrer principal ement des consultations incluant de nouveaux problèmes afin de favoriser l'évaluation du plus large éventail possi ble d'habiletés de communication. Selon Tate et al. ${ }^{59}$, des études aurai ent démontré une fidèlité et une validité satisfaisante pour cette approche. Les critères de performance retenus sont similaires à ceux décrits dans le guide CalgaryCambridge Ilstiennent compte de la situation clinique spécifique, ce qui renforce les liens entre le processus de communication et la démarche clinique («Le médecin obtient suffisamment d'information pour qu'aucune condition sérieuse ne soit manquée ", "Le médecin apparait retenir des hypothèses diagnostiques cliniquement appropriées ${ }^{59}$ ).

Au Q uébec, I'octroi du permis d'exercice de la médecine générale (médecine de famille) fait appel à un processus incluant un examen final à multiples composantes ${ }^{61}$. La qualité des habiletés de communication des futurs médecins y est évaluée en observant leur performance au cours de deux de ses composantes. Au cours de la première, en l'occurrence les différentes stations d'un examen clinique objectif structuré (ECOS), il est possible d'effectuer une évaluation globale (minimale) de certains éléments liés à la structure et au climat de l'entrevue. Cependant, le contexte télescopé de ces stations rend difficile une évaluation approfondie et spécifique des habiletés de communication du candidat.

La deuxième composante de l'examen, les entrevues médicales simulées (EM S), permet une évaluation plus complète des habiletés de communication ${ }^{62}$. D éveloppées par le Collège des médecins de famille du Canada, elles placent les candidats face à cinq situations cliniques différentes (par exemple: hypertension, cancer, infection sexuellement transmissible,...) qui impliquent une dimension psychosociale (par exemple: 
étape spécifique du cycle de la vie, événements stressants; problèmes dans le réseau familial et social) et des défis de communication (par exemple : patient passif, en colère, honteux,$\ldots)^{63}$. La notation se fait selon une grille préétablie et cible, à la fois, la démarche clinique et les habiletés de communication. Chaque entrevue dure 15 minutes. Le rôle des patients y est joué par des médecins de famille. Des efforts importants sont déployés pour bien standardiser tous les éléments de cet examen. Ainsi les scénarios sont acheminés aux médecins-évaluateurs, de façon confidentielle et sécuritaire, trois à quatre semaines avant la tenue de l'examen afin qu'ils puissent se familiariser avec leur rôle. D e plus, au cours de la journée précédant l'examen, les médecinsévaluateurs doivent, sous la supervision d'un médecin coordonnateur, s'exercer à joueur leur rôle et à noter la performance d'étudiants-volontaires (non candidats à l'examen) qui acceptent d'aider à la standardisation. Des contraintes liées à la faisabilité (ressources humaines et monétaires; temps disponible,...) ont amené les responsables à restreindre à cinq le nombre d'entrevues de cette composante de l'examen, ce qui en limite un peu la fidélité qui demeure toutefois acceptable ${ }^{61,62,64}$.

L'évaluation étant un levier puissant pour orienter les apprentissages, les responsables d'examens doivent absolument s'assurer que les habilités requises pour réussir ceux-ci ne sont pas en contradiction avec les élé ments d'une communication efficace. $D$ ans cet ordre d'idée, nous croyons que certains scénarios utilisés lors des ECOS devraient être réécrits parce qu'ils incitent les étudiants à effectuer des entrevues précipitées, très directives, laissant peu ou pas de place aux patients. IIs contribuent au clivage « démarche clinique-communication » discuté précédemment, en suggérant que, pour des problèmes présumés au départ comme étant biologiques, une approche directive, précipitée, centrée sur le médecin est pertinente, acceptable et suffisante, ce qui, répétons-le de nouveau, ne concorde nullement avec les données de la recherche sur les résultats de soins.

II faut souligner enfin l'importance de vérifier que les modalités d'évaluation exigent réellement l'acquisition, par tous les médecins, des habilités de communication nécessaires à la pratique. Pour ce faire, il faut prêter attention à ce que l'expertise en sciences biomédicales et l'expertise en communication fassent, toutes les deux, l'objet d'une évaluation spécifique, la réussite des deux composantes étant obligatoire pour la promotion ou l'accès à l'exercice médical.

\section{La formation des cliniciensenseignants}

On ne peut se le cacher : nombreux sont les cliniciensenseignants ayant besoin de formation sur la communication et sur les méthodes pédagogiques favorisant son apprentissage.

\section{L'enseignant animateur, facilitateur, motivateur}

Les étudiants ou les participants à des sessions de formation apprendront d'autant mieux qu'ils seront motivés par le défi de la solution de problèmes proches de leur réalité clinique actuelle ou future ${ }^{18,65}$. Ceci implique de faire appel, pour les activités d'apprentissage à la communication, à des médecins enseignants respectés dans leur propre discipline clinique et intéressés par la communication. Ces derniers doivent avoir acquis (ou devront acquérir) des habiletés pour l'enseignement en relation individuelle face à face ainsi que pour l'animation de petits groupes. Leurs tâches incluent, entre autres, la planification des moments de formation et la création d'un climat propice aux apprentissages. Ils doivent pouvoir, lors des activités d'apprentissage, centrer l'attention de l'apprenant sur les habiletés spécifiques à acquérir et favoriser une analyse constructive des entrevues observées et des jeux de rôle réalisés. Ils doivent être capables d'assurer une rétroaction spécifique sur les habiletés de communication pratiquées. Les facultés de médecine et les milieux cliniques d'enseignement ont la responsabilité de favoriser et de soutenir le développement professoral, dans le champ de la communication, d'un nombre suffisant d'intervenants de différentes disciplines. Ils doivent susciter et faciliter l'émergence de «champions » de la communication, c'est-à-dire de médecins respectés dans leur spécialité, possédant de bonnes habiletés de communication et choisissant de consacrer du temps et de l'énergie à l'amélioration de la formation à la communication au sein de leur institution et dans leur propre discipline. Nous utilisons ici le terme de «champions » car, à notre avis, il symbolise bien l'énergie et l'ardeur qu'il sera probablement nécessaire de déployer pour relever cet important défi. 


\section{Références}

\section{Les autres cliniciens}

Par ailleurs, comme nous l'avons mentionné précédemment, sil y a des discordances entre les comportements observés chez les cliniciens et les messages adressés aux étudiants, ces derniers retiendront ce que font leurs « modèles » plutôt que les recommandations verbales ou écrites. Or, la plupart des médecins n'ont pas reçu de formation spécifique à la communication. En outre, il est capital de rappeler que les habiletés d'entrevue ne s'améliorent pas sur la seule base de l'expérience. Ainsi, plusieurs des médecins qui supervisent ou côtoient les étudiants et les résidents ne possèdent ni le référentiel, ni le vocabulaire, ni l'expertise pédagogique pour guider leurs échanges avec les étudiants concernant la qualité des entrevues. Cette situation est un défi de taille pour les milieux académiques et cliniques ainsi que pour les associations professionnelles, qui devront se concerter pour mettre en place des stratégies à multiples volets visant l'acquisition ou la consolidation, par tous les cliniciens, des habilités de communication nécessaire à une pratique médicale de qualité

\section{La nécessité d'une stratégie globale}

Apporter des changements dans une organisation peut s'avérer très difficile, compte tenu des intérêts et des objectifs variés qui modulent la motivation des diffé rents acteurs ${ }^{66}$. Chacun d'entre eux, pour modifier son attitude et adhérer à une nouveauté, devra en percevoir le bien fondé et I'utilité potentielle pour sa vie professionnelle et personnelle. Plusieurs auteurs ont proposé différentes approches pour instaurer un changement organisationnel. John Kotter ${ }^{67}$, professeur en leadership à la $\mathrm{H}$ avard Business School, a souligné les étapes essentielles pour la réussite d'un changement. En tenant compte des suggestions de cet auteur, il est licite de recommander, d'abord et avant tout, un support franc et soutenu des directions des facultés de médecine et l'adoption d'une vision institutionnelle claire et motivante de ce que doit être la performance en communication des médecins. L'autre étape est la création d'un groupe de travail, incluant des «champions » de la communication et des membres «forts » de la direction pour guider et donner de la puissance à la démarche. Ce groupe de travail, avec l'appui des directions, doit élaborer un plan d'action... et surtout s'assurer de son implantation ! Un élément essentiel de ce plan et de la réussite du changement sera de chercher à faire ressen- tir par tous l'urgence d'agir. Un autre point incontournable (mais souvent négligé) est d'aligner les incitatifs symboliques (promotion, titre, honneur...) et moné taires (recrutement, rémunération, financement...) avec la vision retenue et le plan d'action décidé. Trop souvent, les beaux discours sont contredits par les critères de promotion ou les décisions budgétaires. Enfin, notons que les progrès surviennent rarement par hasard : il est donc impératif de ne pas attendre et d'être proactifs!

\section{Conclusion}

La société réclame des médecins plus humains et communiquant mieux avec les patients. Les cliniciens expé rimentés et les chercheurs des domaines de l'éducation médicale et de la communication soulignent le rôle central de la communication médecin-patient dans la démarche clinique pour le succès des stratégies de diagnostic et de traitement. Les organismes d'accréditation de plusieurs pays exigent une amélioration majeure des habiletés de communication des médecins. Les travaux des décennies récentes ont permis d'identifier les facteurs-clefs d'une communication efficace et les éléments favorisant l'apprentissage des habiletés requises. Le choix des moyens d'apprentissage doit tenir compte du résultat des recherches en éducation sur les stratégies efficaces (référentiel solide, démonstration, pratique et rétroaction immédiate, évaluation spécifique, activités de connaissance de soi, présence de modèles de rôle, cursus longitudinal). Les travaux du groupe $\mathrm{C}$ al gary-C ambridge sont proposés comme référentiel de base car ils satisfont toutes ces exigences et sont d'une clarté exemplaire.

Bien que beaucoup de progrès restent à faire, plusieurs milieux académiques, surtout anglo-saxons, offrent actuellement des formations à la communication qui, en accord avec les orientations proposées par l'équipe de Calgary-C ambridge, mettent l'accent sur l'acquisition d'habiletés spécifiques, validée à l'aide d'un guide de comportements observables. Toutefois, le temps alloué à l'apprentissage de la communication médecinpatient est souvent insuffisant et son renforcement, au moment de l'internat ou du résidanat, est nettement déficient, y compris dans notre réseau académique montréalais. Par ailleurs, plusieurs autres milieux, notamment francophones, considèrent comme suffi- 
sant, en termes de formation à la communication, I'organisation de discussions sur les aspects affectifs de la relation médecin-patient, ce qui, tout en étant nécessaire, est notoirement insuffisant.

Les facultés de médecine ont la responsabilité de former des médecins aptes à comprendre et à faciliter la résolution des problèmes de santé des citoyens et des citoyennes de leurs pays respectifs. Pour ce faire, les médecins doivent acquérir de solides compétences professionnelles, dont celle incontournable de la communication. Aussi, est-il essentiel et urgent de mettre en place dans chacun de nos milieux les stratégies propres à relever le défi de l'apprentissage de la communication par les médecins.

\section{Remerciements}

Les auteurs remercient très chaleureusement le docteur Suzanne Kurtz et ses collègues pour avoir permis la traduction, I'adaptation et I'utilisation en français de certains des schémas et textes qu'ils avai ent produits, notamment le précieux « Calgary-Cambridge Guide to the M edical Interview - Communication Process ${ }^{37}$. IIs tiennent également à remercier les Éditions du Renouveau Pédagogie pour leur avoir permis de rendre publique la traduction en français du guide Calgary-Cambridge avant sa publication dans « La communication professionnelle en santé $»$, un livre actuellement sous presse ${ }^{68}$.

\section{Annexe 1 : Extrait du Guide Calgary-Cambridge de l'entrevue médicale ${ }^{37,68}$}

\section{I - Débuter l'entrevue}

\section{A - Préparer l'entrevue}

\section{B - Établir le premier contact (l'accueil)}

1. Le médecin salue le patient et obtient son nom.

2. Se présente et précise son rôle, la nature de l'entrevue; obtient le consentement du patient, si nécessaire.

3. Montre du respect et de l'intérêt ; veille au confort physique du patient (du début à la fin de l'entrevue).

\section{C - Identifier la (les) raison(s) de consultation}

1. Identifie, par une question adéquate d'ouverture, les problèmes ou préoccupations que le patient souhaite voir aborder durant l'entrevue ( "Q uels problèmes vous amènent aujourd'hui ? » ou « Q u'est-ce que vous souhaiteriez discuter aujourd'hui ?»)

2. Écoute attentivement les énoncés de départ du patient, sans l'interrompre ou diriger (orienter) sa réponse

3. Confirme la liste initiale des raisons de consultation et vérifie sil y a d'autres problèmes ( $D$ onc, il y a les maux de tête et la fatigue. Y a-t-il autre chose dont vous aimeriez parler aujourd'hui ? »)

4. Fixe, avec l'accord du patient, l'agenda de la rencontre en tenant compte, à la fois, des besoins de ce dernier et des priorités cliniques 


\section{Références}

\section{Références}

1. World $\mathrm{H}$ ealth O rganization. D octor-patient interaction and communication. Genève : Division of $M$ ental Health, 1993.

2. Royal College of Physicians and Surgeons of Canada. Skills for the $\mathrm{N}$ ew Millennium : Report of the Socieal Needs Ottawa : Working Group CanM EDS 2000 Project (Canadian Medical Education Directions for Specialists 2000 Project), 1996.

3. Participants in the Bayer-Fetzer Conference on Physician-Patient Communication in Medical Education. Essential Elements of Communication in Medical Encounters: The Kalamazoo Consensus Statement. Acad M ed $2001 ; 76$ : 390-393.

4. Association of American Medical Colleges Report 3 : Contemporary Issues in M edicine. Communication in medicine. Washington D C : AAM C, 1999.

5. Cohen-Cole SA. The medical interview: the thre function approach. St-Louis (MI) : M osby Year Book, 1991.

6. Simpson M, Buckman R, Stewart M , M aguire P, Lipkin $M, N$ ovack $D, T$ ill J. D octor-patient communication : the Toronto consensus statement. BM J $1991 ; 303$ : 1385-1387.

7. Lipkin M, Putnam SM, Lazare A. The medical Interview. Clinical Care, Education and Research. Frontiers of Primary Care. N ew York : Springer-Verlag, 1995.

8. Silverman J, Kurtz S, Draper J. Skills for communicating with patients. Abingdon (UK) : Radcliffe M edical Press, 1998.

9. Cole SA, Bird J. The Medical Interview. The Thre Function Approach (2nd edition). St Louis (M I): M osby Year Book, 2000.

10. Kurtz S, Silverman J, D raper J. Teaching and Learning Communication Skills in M edicine. Abingdon (UK) : Raddliffe M edical Press 1998.

11. Kurtz S. Doctor-Patient Communication : Principles and Practices Can J N eurol Sci 2002 ; 29 (suppl. 2) : S23-S29.
12. Kurtz S, Silverman J, Benson J, D raper J. M arrying Content and Process in Clinical Method Teaching: Enhancing the Calgary-Cambridge Guides Acad M ed $2003 ; 78: 802-9$.

13. Evidence Based M edicine Working Group. Evidence based medicine A new approach to teaching the practice of medicine JAM A $1992 ; 268: 2420-2425$.

14. Sackett DL, Straus SE, Richardson WS, Rosenberg W, $H$ aynes RB. Evidence-based M edicine: $H$ ow to Practise and Teach EBM (Second Edition). Edinburgh (UK) : Churchill Livingstone, 2000.

15. Van Der Vleuten $C$, D olmans $D$, Scherpbier $A$. The need for evidence in education. M ed Teach $2000 ; 22$ : 246-250.

16. Jean P. Pour une planification méthodique des activités d'apprentissage. Pédagogiemédicale 2001 ; 2 : 101-107.

17. Epstein R, Campbell T, Cohen-Cole S, M cWinney I, Smilkstein G. Perspectives on Patient-D octor Communication. J Fam Pract $1993 ; 37$ : 377-388.

18. Tardif J. Pour un ensegnement stratégique L'apport de la psychologie cognitive M ontréal (QC) : Les Éditions Logiques, 1992 (réédition 1997).

19. Schmidt, H.G. \& M oust, J.H.C.. Factors Affecting Small-Group Tutorial Learning: A Review of Research. In Evensen, D orothy $H \& H$ melo, Cindy (eds.) Problem-Based Learning. A Research Perspective on Learning Interactions. Mahwah (NJ) : Lawrence Erlbaum Associates, 2000 : 19-52.

20. Balint M . Lemédean, son maladeet la maladie. Paris: Petite bibliothèque Payot, 1966.

21. Jean $P, D$ esM archaisJE, D elormeP. Apprendreà enseigner les sciences de la santé, Guide de formation. Cahier 3. M ontréal et Sherbrooke : Faculté de médecine des universités de M ontréal et de Sherbrooke (4 édition), 1993.

22. Smith RC, Lyles JS, Mettler J, Stoffelmayr $B E$, Van Egeren, LF, M arshall, AA, Gardiner JC, M aduschke KM, Stanley JM, O soorn GG, ShebroeV, Greenbaum $R B$. The effectiveness of intensive training for residents in Interviewing: A randomized, controlled study. Ann Intern M ed 1998 ; 128 :118-126. 
23. Aspegren K. BEM E Guide No 2: Teaching and learning communication skillsin medicine- a review with quality grading of artides. M ed Teach $1999 ; 21$ : 563570 .

24. Fellowes $D$, Wilkinson $S, M$ oore $P$. Communication skills training for health care professionals working with cancer patients, their families and/or carers Cochrane D atabase of Systematic Reviews 2003 (3).

25. Chan C, Wun Y, Cheung A, Dickinson J, Chan K, Le $H$, Yung $Y$. Communication skill of general practitioners any room for improvement? How much can it be improved? M ed Educ 2003 ; 37 : 514-526.

26. Eptein R. Mindful Practice JAMA 1999 ; 282 : 833-859.

27. M aguire $P$, Pitceathly $C$. Key communication skills and how to acquire them. BMJ $2002 ; 325: 697-700$.

28. Enelow AJ, Swisher SN. Interviewing \& Patient Care (Second Edition). New-Y ork (USA) / O xford (UK) : O xford U niversity Press, 1979.

29. Pendleton $D$, Schofield $T$, Tate $P, H$ avelock $T$. The Consultation, an Approach to Learning and Teaching. Oxford (UK) : O xford U niversity Press, 1984.

30. Coulehan JL, Block MR. The M edical Interview : A Primer for Students of theArt. Philadelphie (PE) : FA Davis Company, 1987.

31. Billings JA, Stoeckle JD. The Clinical Encounter, a Guide to the M edical Interview and Case Presentation. Chicago (M I) : Year Book M edical Publisher Inc, 1989.

32. Stewart $M$, Roter D. Communicating with M edical Patients. N ewbury Park (CA) : Sage Publication Inc., 1989.

33. Stewart M, BelleBrown J, Weston WW, M CWinney IR, $M$ CWilliam CL, Freeman TR. Patient-C entered Medicine, Transforming the Clinical Method, Second edition. Abingdon (UK) : Raddliffe Medical Press, 2003.

34. Bird J, Cohen-ColeSA. Thethree function model of the medical interview: an educational device. In : $\mathrm{H}$ ale $\mathrm{M}$ (ed). Models of consultation-liaison psychiatry. Basel (Switzerland) : Karger, 1990.
35. Lazare A, Putnam SM, Lipkin M Jr. Three functions of the medical interview. In : Lipkin M, Putnam, SM, Lazare A. The Medical Interview. Clinical Care, Education, and Research. $\mathrm{N}$ ew York: Springer-V erlag, 1995 : 3-19.

36. Bensing J. B ridging the gap. The separate worlds of evidence-based medicine and patient-centered medicine Patient Education and Counseling $2000 ; 39$ : 17-25.

37. Calgary-Cambridge Guide to communication : Process skills. In : Kurtz SM , Silverman JD, D raper J. Teaching and Learning Communication Skillsin M edicine (2nd edition). Abingdon (UK) : Raddiffe Medical Press (Oxford), 2004 (sous presse) et In : Silverman JD, Kurtz SM, Draper J. Skills for communicating with patients (2nd edition). Abingdon (U-K) : Raddliffe M edical Press (0 xford), 2004 (sous presse).

38. Comité de planification de l'atelier. Protocole d'accord de l'atelier sur l'ensegnement et l'évaluation des techniques de communication dans les facultés de médecine Can Med AssJ 1992 ; 147 : 1151-1152.

39. General Medical Council. Tomorrow's Doctors : Recommendations on Undergraduate M edical education. London: GM C 1993.

40. Miller GE. The assessment of clinical skills/competencelperformance Acad M ed $1990 ; 65$ : 63S - 67S.

41. Bensaid N. La consultation : le dialogue médecin/malade Paris: Bibliothèque $M$ édiations, D enö̈/G onthier, 1979 : 236.

42. Novack D, Suchman A, Clark W, Epstein R, N ajberg E, Kaplan C. Calibrating the Physician: Personal Awareness and Effective Patient Care. JAM A 1997 ; $278: 502-509$.

43. Selvini-Palazzoli M, Boscolo L, Ceechin G, Prata, G. $H$ ypothesizing, circularity, neutrality: Three guidelines for the conductor of the session. Fam Process $1980 ; 19$ : 3-12.

44. N ovack D, Epstein R., Paulsen R. Toward Creating Physician-Healers Fostering Medical Student's Selfawareness Personal Growth, and Well-being. Acad M ed $1999 ; 74: 516-520$.

45. M oreau Ricaud M. Le groupe Balint a cinquante ans Topique $2001 ; 76: 93-102$. 


\section{Références}

46. Johnston $A H, B$ rock $C D, H$ amadeh $G$, Stock R. The Current Status of Balint Groups in American Family Practice Reidencies : a Ten Year Follow-up Study, 1990-2000. J Balint Soc $2001 ; 29$ : 11-17.

47. Hafferty F, Franks R. TheH idden Curriculum, Ethics Teaching, and the Structure of Medical Education. Acad M ed 1994 ; 69 : 861-871.

48. Williams $C, C$ antillon $P$, Cochrane M. The doctorpatient relationship: from undergraduate assumptions to pre registration reality. M ed Educ $2001 ; 35$ : 743-747.

49. Schutzenberger A. Introduction au jeu derôle. Toulouse (France): Privat, 1975.

50. Steinert $Y$. Twelvetips for using roleplay in clinical teaching. M ed Teach1993 ; 15 : 283-291.

51. Ende J. Feedback in Medical Education. JAMA $1983 ; 250: 777-781$.

52. O'Brien H V, M arks M B, Charlin B. Le feedback (ou rétro-action) : un éément essentiel de l'intervention pédagogique en milieu clinique Pédagogie M édicale $2003 ; 4: 184-191$.

53. Steinert $Y$. Twelve tips for using videotape reviews for feedback on dinical performance. Med Teach 1993 ; 15 : 131-139.

54. Sanson-Fisher R, Cockburn J. Effective teaching of communication skillsfor medical practice: selecting an appropriate clinical context. M ed Educ 1997 ; 31 : 52-57.

55. Hodges $B$, Turnbull J, Cohen R, Bienenstock $A$, Norman $G$. Evaluating communication skills in the objective structured clinical examination format: reliability and generalizability. Med Educ $1996 ; 30$ : 3843.

56. Boon $\mathrm{H}$, Stewart M. Patient-physi dian communication assessment instruments: 1986 to 1996 in review. Patient Education and Counseling 1998 ; 35 : 161176.

57. M akoul G, Schofield T. Communication teaching and assessment in medical education: an international consensus statement. Patient education and Counseling 1999 ; $137:$ 191-195.
58. M akoul G. The SEGUE Framework for teaching and assessing communication skills. Patient Education and Counseling $2001 ; 45: 23-34$.

59. Tate $P$, Foulkes J, N eighbour $R$, Campion $P$, Field $S$. Assessing Physicianś Interpersonal Skills via Videotaped Encounters. A N ew Approach for the Royal College of General Practitioners Membership Examination. J H ealth Communication $1999 ; 4$ : 143-152.

60. Royal College of General Practioners (RCGP). M embership RCG P examination. London, UK, 2003 ; < http://www.rcgp.org.uk/exam/index.asp >.

61. Grand'M aison P, Brailovsky CA, Lescop J, Rainsberry P. $U$ sing Standardized Patients in Licensing/C ertification Examinations Comparison of Two Tests in Canada. Fam M ed $1997 ; 29: 27-32$.

62. Handfield-Jones R, Belle Brown J, Rainsberry $P$, Brailovsky CA. Certification Examination of the College of Family Physicians of Canada. Part 2. Conduct and general performance. Can Fam Physician 1996 ; 42 : 1188-1195.

63. Collège desmédecins de famille du Canada. Examenset certification. Toronto (ON) 2003 < http://www. ffpc.ca/global/splash/default.asp?s=1 >.

64. Handfield-Jones R, Rainsberry P. Certification Examination of the College of Family Physicians of Canada. Part 1. History and implications for the pre sent and future. Can Fam Physician 1996 ; 42 : 957969.

65. Norman G, Schmidt H. The Psychological Basis of Problem-based Learning: A Review of Evidence. Acad M ed $1992 ; 67$ : 557-565.

66. Guilbert JJ. Vers une formation pertinente des personnels de santé. Pourquoi cela prend-t-il du temps? Q ue faire ? Pédagogie M édicale 2003 ; 4 : 35-41.

67. Kotter JP. Leading Change. Boston : $\mathrm{H}$ avard Business School Press, 1996.

68. Richard C, Lussier MT (éditeurs). La communication professionnelle en santé M ontréal, Canada : Les Éditions du Renouveaux Pédagogiques Inc., 2004 (sous presse). 